\title{
Effect and Molecular Mechanisms of Traditional Chinese Medicine on Tumor Targeting Tumor-Associated Macrophages
}

This article was published in the following Dove Press journal:

Drug Design, Development and Therapy

Jing $\mathrm{He} \mathbb{1 D}^{\prime}$

Peihao Yin ${ }^{1-4}$

$\mathrm{Ke} X \mathrm{u}^{\mathrm{I}, 3,4}$

'Putuo Hospital, Shanghai University of Traditional Chinese Medicine, Shanghai, People's Republic of China; ${ }^{2}$ Department of General Surgery, Putuo Hospital, Shanghai University of Traditional Chinese Medicine, Shanghai, People's Republic of China; ${ }^{3}$ Shanghai Putuo Central School of Clinical Medicine, Anhui Medicine University, Anhui, People's Republic of China; ${ }^{4}$ Interventional Cancer Institute of Chinese Integrative Medicine, Shanghai University of Traditional Chinese

Medicine, Shanghai, People's Republic of China
Correspondence: Peihao Yin; Ke Xu Putuo Hospital, Shanghai University of Traditional Chinese Medicine, Shanghai, People's Republic of China Email yinpeihao@shutcm.edu.cn; kexu2577@shutcm.edu.cn

\begin{abstract}
Traditional Chinese medicine (TCM) has been used as a significant cancer treatment method for many years in China. It has been demonstrated that TCM could assist in inhibiting the growth of tumors and prolonging the survival rates of cancer patients. Although the mechanism of TCM are still not clear, accumulating evidence has shown that they may be related to the tumor microenvironment (TME). Tumor-associated macrophages (TAMs) play a significant role in TME and are polarized to two phenotypes, M1 (classically activated) and M2 (alternatively activated) TAMs. The two different phenotypes of TAMs play converse roles in the TME and M2-polarized tumor-associated macrophages (M2TAMs) always lead to poor prognosis in cancer patients compared to M1-polarized tumorassociated macrophages (M1-TAMs). In this review, the potential correlation between TCM and TAMs (especially the M2 phenotype) in tumor progression and promising TCM strategies targeting TAMs in cancer are discussed.
\end{abstract}

Keywords: traditional Chinese medicine, TCM, tumor-associated macrophages, TAMs, tumor microenvironment

\section{Introduction}

Traditional Chinese medicine (TCM) has been used as a very important tumor treatment strategy for many years in China. ${ }^{1}$ TCMs and their active ingredients have been shown to enhance the antitumor effects and reduce the toxicity of chemotherapy and radiotherapy, alleviate the symptoms of cancer, and prolong the survival rates of cancer patients in many clinical and preclinical studies. ${ }^{2-11}$ Although it is not clear how TCM plays a role in tumors, increasing evidence has shown that the mechanism of TCM may be related to its synergistic effect on regulating the tumor microenvironment (TME). ${ }^{12,13}$

Among tumor-infiltrating immune cells, tumor-associated macrophages (TAMs) constitute an important population, and considerable data have indicated that TAM infiltration into tumors always leads to poor prognosis. ${ }^{14-17}$ In addition, TAMs are stimulated by different molecules to polarize into two phenotypes, classically activated phenotype (M1) and alternatively activated phenotype (M2) TAMs. The two phenotypes of TAMs, M1-polarized tumor-associated macrophages (M1TAMs) and M2-polarized tumor-associated macrophages (M2-TAMs), play important roles in the TME by functioning as immune cells and playing different roles in cancer cells. Furthermore, M2-TAMs could diminish effective antitumor immune 
responses, promote angiogenesis and cause vascular permeability to support tumor growth. Therefore, TAMtargeted therapy, including the functional suppression of M2-TAMs and repolarization of M2-like TAMs towards the M1-like phenotype, has emerged as a novel and promising strategy for cancer treatment. ${ }^{18}$

In this review, we suggest a hypothesis that the synergistic effect and molecular mechanisms of TCM in cancer treatment are related to TAMs (especially M2-TAMs) in the TME, and we hope to find promising strategies targeting TAMs for tumor treatment using TCMs and their active ingredients.

\section{The Mechanisms of TAMs in Tumors}

Tumor microenvironment (TME), composed of tumor cells and surrounding stroma, is related to the progression and metastasis of tumor, and immunosuppression. ${ }^{19}$ Additionally, one of cancer therapies is remodeling TME. ${ }^{18}$ TAMs are a major component of the tumor microenvironment (TME) as immune regulators and potential targets. ${ }^{14,20,21}$ TAMs are known to be polarized into two phenotypes, M1 (classically activated) and M2 (alternatively activated) TAMs, and these two types play different roles in the TME. ${ }^{18,22}$ Increasing studies have shown that the M1 phenotype exerts tumor resistance effects, while the M2 phenotype promotes tumors in the TME; $;^{18,23}$ both of these functions are related to their roles as immune cells.

\section{The Roles of MI-TAMs and M2-TAMs as Pivotal Immune Cells}

Considering different stages of tumor development, TAMs could enable a dual role by switching two phenotypes as M1-like phenotype and M2-like phenotype. When in earlystage of tumor progression, TAMs mostly display M1 phenotype to cause tumor cell disruption. Conversely, the majority of TAMs show M2 phenotype in tumoral late-stage, followed the decreased antitumoral capacity. During various stages, there is not only one phenotype of TAMs but M1-polarized TAMs and M2-polarized TAMs coexisting. ${ }^{24}$ And the balance of M1 and M2 phenotype determines patients' outcome. The difference between stages is the occupancy rate of two phenotypes TAMs and this rate could be changed by the changeable environment or components, added medicants and various kinds of targets. For example, the removal of apoptotic neutrophils could reverse M1-TAMs to M2-TAMs; ${ }^{25}$ the polarization of TAMs to M2 phenotype could be promoted by tumor hypoxia; ${ }^{26}$ targeting CSF1/CSF1R axis could repolarize the phenotype of TAMs of M2-like to M1-like. ${ }^{27}$ M1-TAMs and M2-TAMs are coexisting and functioning differently in TME, and could repolarize to each other.

The antitumoral capacity of M1-TAMs is always related to the inflammatory response and the activation of specific lymphocytes. Interferon- $\gamma$ (IFN- $\gamma$ ) could induce M1-TAMs alone or cooperate with cytokines (tumor necrosis factor (TNF)- $\alpha$ ) or microbial stimuli (lipopolysaccharide (LPS)) to secrete proinflammatory cytokines, such as (C-X-C motif) ligand 9 (Cxc19), Cxcl10, Cxcl5, TNF- $\alpha$, interleukin (IL)-1, IL-6, IL-12, or IL-23, and exert great phagocytic and microbicidal ability. ${ }^{23,28-34}$ In addition, M1-TAMs contribute to the higher expression levels of major histocompatibility complex class II (MHC II) molecules and higher secretion levels of IL-12; these changes could induce an antiangiogenic effect by increasing the expression of Cxcl10 or IP-10, which is chemokine inducible protein-10, and promote the bactericidal activity of phagocytes through naïve $\mathrm{T}$ cells differentiating into Th1 cells to stimulate the growth of both natural killer (NK) cells and $\mathrm{T}$ cells $\mathrm{s}^{23,24,28,34}$ (Figure 1).

In contrast, M2-TAMs play a role in supporting tumor growth by upregulating anti-inflammatory cytokine expression and suppressing inflammation in the tumor immunosuppressive microenvironment (TIM). M2-TAMs are activated by IL-4 and IL-13, known as Th2 cytokines, to downregulate IL-12 and IL-23 and increase the expression level of IL-10, which is an anti-inflammatory cytokine, and conversely, IL-10 expressed by M2 macrophages stimulates Th2 cells to produce IL-4 and IL-13. ${ }^{23,34-37}$ The activation of IL-4 or IL-13 accompanied with Fizz-1's high expression, which is an indicator of the polarization from TAMs to M2 phenotype. The functions of Fizz-1 in TME include inflammation, angiogenesis, and cell proliferation. ${ }^{38-40}$ In addition, IL-10 could suppress proinflammatory cytokine (including IFN- $\gamma$, IL-2, IL-3, and tumor necrosis factor- $\alpha$ (TNF- $\alpha)$ ) synthesis and inhibit antigen-presenting cells from presenting antigens. Furthermore, M2-TAMs can produce ccl17, ccl22, ccl24, and ccl22 and inhibit CD4+ and CD8 $+\mathrm{T}$ cell effector functions and recruit regulatory $\mathrm{T}$ cells (Tregs) to the $\mathrm{TME}^{18,28,41-44}$ (Figure 1).

M1-TAMs secrete proinflammatory cytokines (TNF- $\alpha$, interleukin (IL)-1, IL-6, IL-12, or IL-23) to exert great phagocytic and microbicidal ability. Additionally, M1TAMs could promote the bactericidal activity of phagocytes by differentiating naïve T cells into Th1 cells to stimulate the growth of both $\mathrm{T}$ and NK cells. M2-TAMs secrete 


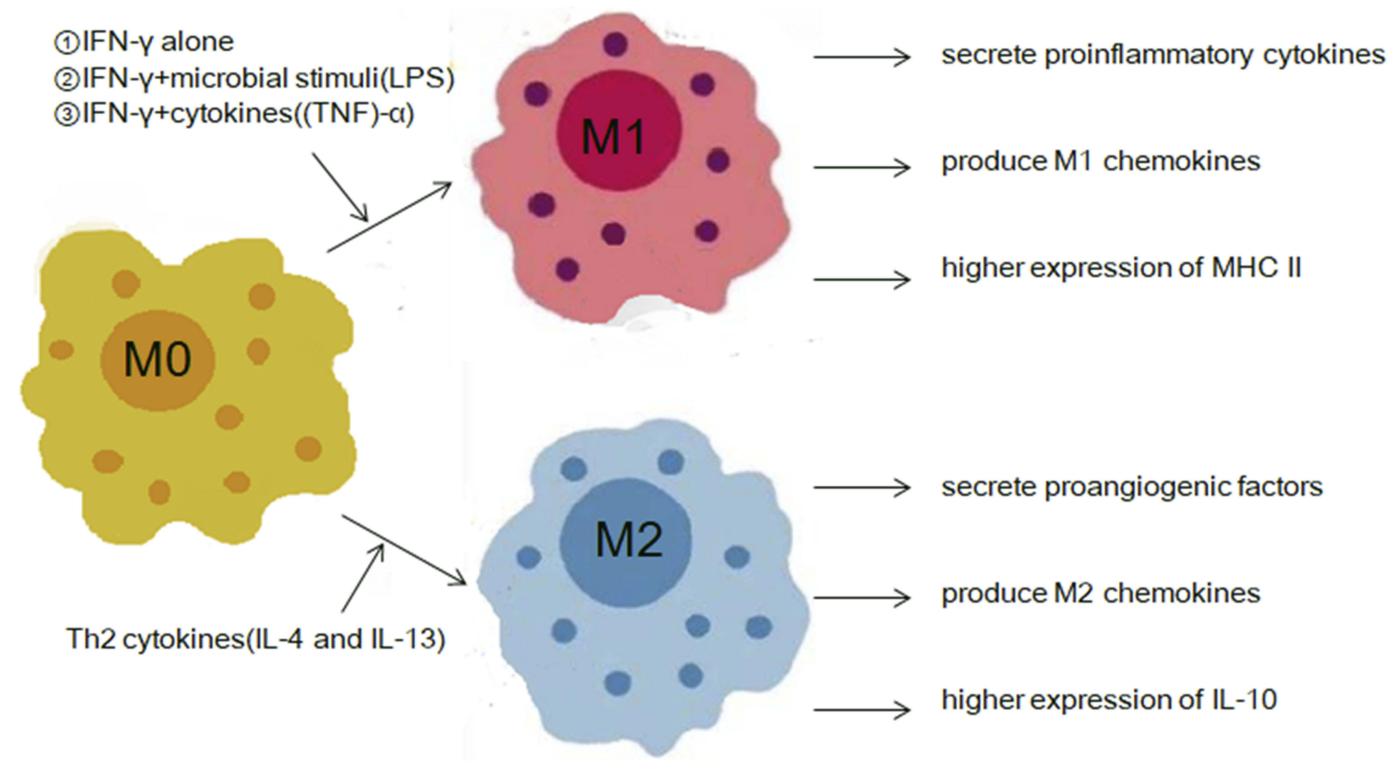

Figure I Polarization of TAMs and the immune roles of the MI phenotype and M2 phenotype in the TME. Abbreviations: TME, tumor microenvironment; TAMs, tumor-associated macrophages.

proangiogenic factors, such as vascular endothelial growth factor (VEGF) and matrix metallopeptidase 9 (MMP9), to support metastasis and tumor growth. M2 cytokines, such as ccl17, ccl22, and ccl24, could inhibit CD4+ and CD8+ T cell effector functions and recruit regulatory $\mathrm{T}$ cells (Tregs) to the TME. In addition, a high level of IL-10 expression suppresses the synthesis of proinflammatory cytokines and inhibits antigen-presenting cells from presenting antigens.

\section{Direct Effects of MI-TAMs and M2-TAMs on Cancer Cells}

As mentioned above, the polarization of TAMs to the M1 phenotype is related to the inflammatory response and the activation of specific lymphocytes, both of which are methods for attempting to eliminate tumor cells. ${ }^{29,33,34}$ Additionally, in the early stage of tumor progression, M1-like phenotype TAMs exert tumor resistance effects by causing tumor cell disruption by expressing high levels of IL-12 $2^{34}$ (Figure 2).

Studies have shown that M2-TAMs could promote cancer progression by supporting the proliferation, migration, and invasion of cancer stem cells. Huang et $\mathrm{al}^{45}$ found that after co-incubating M2-TAMs with A549 and H441 cells, which are non-small cell lung cancer (NSCLC) cell lines, the progression of cancer stem cells was promoted by increased CD133-positive cell populations, increased mRNA levels of Muc-1, CD133 (stemness), and NF-kB (inflammation),

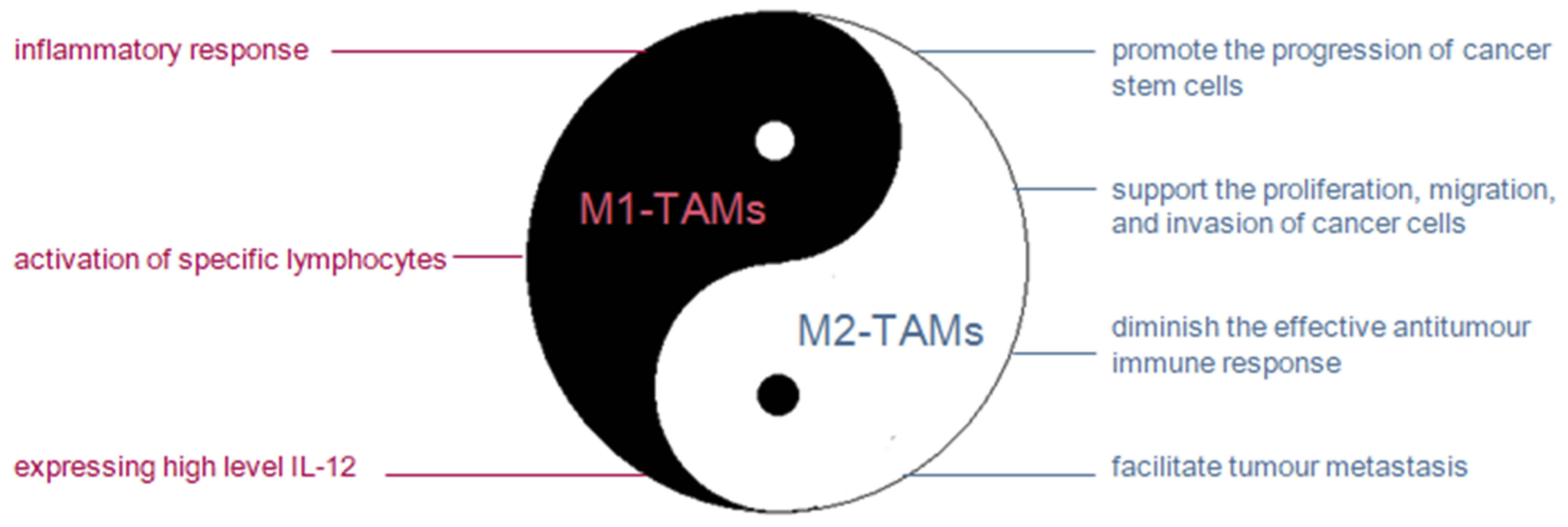

Figure 2 Antitumoral effects of MI-TAMs and protumoral effects of M2-TAMs in the TME.

Abbreviations: TME, tumor microenvironment; M2-TAMs, M2-polarized tumor-associated macrophages; MI-TAMs, MI-polarized tumor-associated macrophages. 
and enhanced self-renewal ability of both NSCLC cell lines. As cancer stem-like cells (CSCs) show resistance to apoptosis and have continuous self-renewal and proliferation capacity, M2-TAMs involved in regulating CSC function could indirectly promote tumor progression. In addition, accumulating evidence has suggested that the polarized-M2 phenotype could increase the proliferation, migration and invasion abilities of cancer cells. ${ }^{18,23,46}$ Zhao et al ${ }^{47-49}$ found that M2TAMs enhanced metastasis by improving the migration capacity of breast cancer $4 \mathrm{~T} 1$ cells. In addition, Pang et al ${ }^{46}$ found that culturing NSCLC cells with M2-polarized TAMconditioned medium (TAM-CM) ultimately led to stronger proliferation, migration, and invasion than culturing these cells in routine medium (Figure 2).

M1-TAMs are involved in the inflammatory response and the activation of specific lymphocytes to eliminate tumor cells. In addition, M1-TAMs could cause tumor cell disruption by inducing high levels of IL-12 expression.

M2-TAMs could promote the development of tumors by promoting the progression of cancer stem cells, supporting the proliferation, migration, invasion of cancer cells, diminishing effective antitumor immune responses, and facilitating tumor metastasis.

\section{M2-TAMs Diminish the Effective Antitumor Immune Response}

Compared to the antitumor effects of M1-TAMs via the inflammatory response, the protumoral roles of M2-TAMs are related to downregulating the effective antitumor immune response by releasing chemokines that play basic roles in immunosuppression and secreting immune suppression molecules, such as transforming growth factor- $\beta$ (TGF- $\beta$ ), IL-10, and arginase-1 (Arg-1), ${ }^{23,25,46,50-53}$ Which enable M2-polarized TAMs to block immune responses against tumor antigens of $\mathrm{T}$ cell. ${ }^{25}$

Chemokines, for example, Ccl13/18/22, are considerable chemoattractants of immune suppressor cells (such as Tregs) that inhibit antitumor immunity to promote the growth of tumors and decrease the survival rate of patients. ${ }^{23,28}$ In addition, $\mathrm{Ccl} 2$ and $\mathrm{Ccl} 5$ have the capacity to suppress T-cell responses. ${ }^{23,28}$

TGF- $\beta$ could block the stimulation, proliferation, differentiation, and effector function of conventional CD4+ and $\mathrm{CD} 8+\mathrm{T}$ cells to inhibit the antitumor response directly. ${ }^{54-57}$ Moreover, TGF- $\beta$ blocks the immune function of conventional $\mathrm{CD} 4+$ and $\mathrm{CD} 8+\mathrm{T}$ cells by promoting the induction of $\mathrm{CD} 4+\mathrm{CD} 25+\mathrm{FoxP} 3+$ regulatory T cells. ${ }^{58-60}$ TGF- $\beta$ also inhibits the cytolytic activity of NK cells ${ }^{56,61}$ and maintains Treg cell differentiation ${ }^{57}$ to suppress the antitumor response.

Like TGF- $\beta$, IL-10 also has the ability to block the function of conventional CD4+ and CD8+ T cells to significantly reduce the development of effector T cells. ${ }^{62-64}$ In addition, IL-10 inhibits antitumor immunity by impeding the production of IL-12, releasing the cytokine IFN- $\gamma$, and blocking epidermal antigen presenting cells (APCs) from presenting tumor-associated antigens. ${ }^{65,66}$

Furthermore, IL-10 and TGF- $\beta$ could revert immune cells to immunosuppressive phenotypes, such as regulatory $\mathrm{T}$ cells, regulatory $\mathrm{B}$ cells, and even TAMs. These cells express immunosuppression and protumor cytokines, consume proinflammatory factors (IL-2, TNF $\alpha$ ), and promote angiogenesis and tumor invasion by producing matrix metalloproteinases (MMPs) and vascular endothelial growth factor (VEGF). The vicious cycle of TAMs, IL10 and TGF- $\beta$ results in poor prognosis in cancer patients. ${ }^{67-70}$

Arg-1 suppresses antitumor immunity with its immunosuppressive catabolic products ${ }^{71-74}$ and blocks the ability of $\mathrm{T}$ cells to generate immune effector cells by exhausting arginine from the environment of conventional $\mathrm{T}$ cells. ${ }^{71-74}$ In addition, high Arg-1 expression might downregulate NO-regulated tumor cytotoxicity, increase the proliferation of cells, dysregulate $\mathrm{T}$ cell receptor (TCR) signaling, and subsequently induce CD8+ T cell unresponsiveness to promote tumor growth. ${ }^{75,76}$

\section{M2-TAMs Facilitate Tumor Metastasis}

To function in tumor metastasis, M2-like TAMs produce VEGF and type IV collagenases MMP2 and MMP9, which promote not only angiogenesis in tumor progression but also tumor migration by causing vascular permeability. ${ }^{18,77-86}$ Additionally, TAMs indirectly upregulate the expression of proangiogenic factors (such as Cxcl12), and Cxcl12 could promote M2 macrophage polarization as a chemoattractant of macrophages in prostate cancer. ${ }^{18,23}$ However, the correlation between M2-TAMs and proangiogenic factors requires more investigation. In addition to promote angiogenesis, TAMs also promote lymphangiogenesis in tumor by transdifferentiate as endothelial progenitor cells. ${ }^{87}$

Besides, epithelial-mesenchymal transition (EMT) is a pivotal step in tumor invasion and migration. M2-like TAMs upregulate IL-10 production by activating Toll-like 
receptor 4 (TLR4) to enhance EMT in pancreatic cancer cells, ${ }^{88}$ and they activate the epidermal growth factor receptor (EGFR) pathway by inducing epidermal growth factor-like (EGF-like) ligand secretion in lung cancer cells. $^{89}$ Both of these factors could ultimately promote EMT and result in facilitating tumor metastasis. ${ }^{18}$

Studies have shown that TAMs secrete MMPs (such as MMP-2, 7, and 9) and subsequently induce the expression of Vascular endothelial growth factor C (VEGF-C), which stimulates the formation of lymphatic vessels to promote tumor metastasis. ${ }^{90}$

\section{Correlation Between TCM and TAMs}

The significant advantage of Western medicine in cancer treatment is its focus on definite targets, such as monoclonal antibodies against cancer cells or immunosuppressive factors, to suppress tumor progression. However, cixutumumab-associated dermatologic events suggest underlying problems and side effects of Western medicine. Introducing traditional Chinese medicine (TCM) not only counteracts the shortcomings of Western medicine but also helps prolong the survival rates of cancer patients. TCM treatment has been demonstrated to increase the cancer survival rate, ${ }^{91}$ and the effects of TCM treatment on tumors could be observed in the development of tumors, the activation of cancer cells and the expression levels of tumor-associated molecules. At the oncological level, TCM could inhibit the progression of tumors to increase the survival rate of tumor patients by decreasing tumor weights and volumes and suppressing tumor generation. ${ }^{45,46,92,93}$ From the perspective of cancer cells, TCM has the ability to promote cancer cells to apoptosis and suppress the actions of cancer cells, such as proliferation, migration, invasion, and even viability. ${ }^{46,49,91-96}$ An increasing number of investigations have suggested that TCMs can reduce the expression level of oncogenic proteins and tumorrelated mRNAs at the molecular level. ${ }^{45,46,91,94,95,97-100}$

In addition, evidence has shown the important role of TAMs in the tumor immunosuppressive microenvironment (TIM), and the two polarized phenotypes are related to different effects on tumors. Here, we describe the potential roles of TCM in the TIM and suggest a hypothesis that the antitumor ability of TCM treatment is related to M1polarized TAMs and M2-polarized TAMs.

\section{The Effects of TCM Treatment on the TIM}

Many studies have demonstrated that chronic inflammation has an important functional effect on the tumor microenvironment. Immune cells constitute a part of the tumor immunosuppressive microenvironment (TIM), which is related to immunologic function, angiogenesis and lymphangiogenesis in tumors, and could promote tumor progression. Therefore, researchers have regarded the TIM as a promising target for tumor treatment and have thought TCM treatment could potentially enhance tumor immune responses in the TIM. ${ }^{101}$ MHC class I molecules have the capacity to kill tumor cells by activating cytotoxic lymphocytes (CTLs) and launching a sequence of cytolysis reactions; MHC class II molecules have the ability to induce a cellular-mediated immune response by presenting tumor antigens to CD4+T helper cells. However, immune cells and malignant cells in the TIM downregulate the expression of MHC class I molecules and upregulate the expression of nonclassical human leukocyte antigen (HLA), which is related to poor prognosis in cancer patients. Studies have proven that TCM, eg, Invigorating Spleen and Detoxification Decoction (ISD), can enhance the expression of both MHC class I and MHC class II molecules to promote the immune response. ${ }^{102} \mathrm{TCM}$ induces apoptosis in tumor cells via the Fas/FasL pathway, which is known as an important immune regulatory pathway. FasL overexpression is related to the promotion of tumor cell immune escape, and tumor cells rarely express Fas or express nonfunctional Fas. ${ }^{103}$ The TCM treatment Yang Wei Kang Liu Granule (YWKL) has the ability to increase the expression level of FasL and reduce the expression level of Fas, which results in tumor cell apoptosis. Cancer stem-like cells (CSCs) participate in promoting the formation of the TIM and prevent antitumor responses because they express low quantities of immune recognition molecules and costimulatory molecules. Investigations have shown that TCM may attenuate the oncogenicity of CSCs; for example, bufalin could inhibit the proliferation of CSCs, ${ }^{104}$ and Huaier (Trametes robiniophila Murr.) aqueous extract could downregulate the Wnt/ $\beta$ catenin pathway to inhibit the self-renewal of CSCs. ${ }^{105}$

\section{Effect of TCM Treatment on MI-TAMs}

It is widely known that M1-polarized TAMs play an antitumoral role in the TME, while TCM treatment inhibits the progression of tumors. Furthermore, TCMs have the ability to enhance the anti-tumor effects of M1-polarized TAMs by 
increasing M1 polarization (Figure 3), which could be detected by the upregulated expression of M1-specific markers or the mRNA expression of M1-related molecules.

Polyporus polysaccharide (PPS) is extracted from Polyporus, a TCM anti-tumoral and immunoregulatory medicinal fungus. Liu et al used reverse transcription polymerase chain reaction (RT-PCR) and found that PPS increased the mRNA levels of inducible nitric oxide synthase (iNOS), TNF- $\alpha$ and IL-6, which were related to M1-TAMs. Additionally, compared with that in untreated RAW264.7 cells, the expression of CD86 in PPS-treated cells was increased by $66.0 \%$, which suggested that PPS could promote the polarization of the M1 phenotype of TAMs. ${ }^{106}$

The Qing-Re-Huo-Xue (QRHX) formula is a traditional Chinese formulation consisting of Scutellaria baicalensis and Radix Paeoniae Rubra. An investigation found that QRHX treatment in a Lewis lung cancer (LLC) mouse model resulted in increased mRNA expression levels of
iNOS, an M1 marker, and decreased mRNA expression levels of Arg-1, an M2 marker. In addition, many TCMs, such as berberine and G-Rh2, have the same effect as QRHX on tumor cells, but whether the mechanism of TCM is reversing the M2 phenotype to the M1 phenotype or blocking polarization into the M2 phenotype while promoting M1 polarization remains unclear. ${ }^{107}$

\section{TCM Treatment Decreases the Population of M2-TAMs and Blocks the Polarization of TAMs into the M2 Phenotype}

TCM treatment has four methods for reducing M2-TAMs: decreasing the population of M2-TAMs, blocking the polarization of TAMs into M2-TAMs, suppressing the functional roles of M2-TAMs, and converting the M2 phenotype to the M1 phenotype (Figure 3).

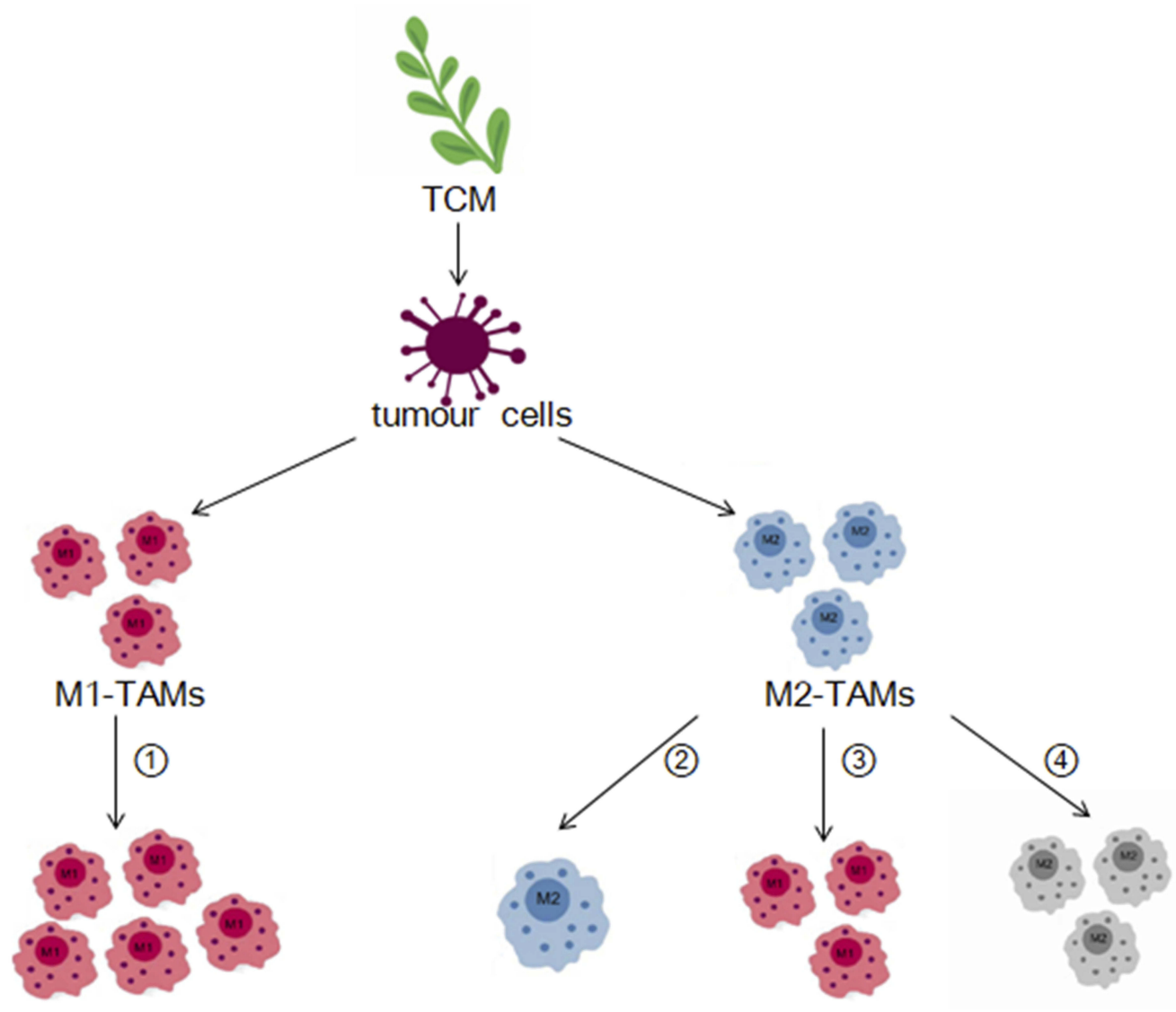

Figure 3 Roles of TCM in MI-TAMs and M2-TAMs.

Notes: TCM have effects on tumor cells by targeting MI phenotype and M2 phenotype TAMs. (1) TCM treatment could increase the population of MI-TAMs through increasing MI polarization. (2) Number of M2-TAMs decrease because TCM block the polarization of TAMs to M2 phenotype. (3) TCM reverse the phenotype of TAMs from M2 to MI. (4) Monochrome M2-TAMs means the protumoral capacities of M2-TAMs are suppressed.

Abbreviations: TCM, traditional Chinese medicine; TAMs, tumor-associated macrophages; M2-TAMs, M2-polarized tumor-associated macrophages; MI-TAMs, MIpolarized tumor-associated macrophages. 
Total flavonoids from TFRG Glycyrrhiza Radix et Rhizoma (Glycyrrhiza Radix et Rhizoma), a vital extract from Gancao (Glycyrrhiza Radix et Rhizoma), have been demonstrated to block signal transducer and activator of transcription 6 (STAT6) activation induced by IL-4/IL-13 by reducing the level of phosphorylated STAT6. Additionally, studies have shown that miR-155 is related to the polarization of TAMs to the M2 phenotype. ${ }^{108}$ The induction of IL-4/IL-13 led to the downregulation of miR155, and TFRG pretreatment significantly increased the level of miR-155. This evidence suggests that TFRG may block M2 polarization by the STAT6 signaling pathway and miR-155. ${ }^{48}$

Wang et al found obvious decreases in the population of M2-TAMs in the spleens of mice treated with osthole. ${ }^{93}$ Using a Bayesian model to analyze the mRNA expression levels of M1- or M2-associated genes, the results proved that PHY906 could promote the polarization of TAMs into the M1 phenotype, improving sorafenib (So) tumor cell treatment, which means that the number of M2-TAMs was lower in the So + PHY906 treatment group than in the sorafenib only treatment group. ${ }^{92}$

Decreased expression levels of specific mRNAs associated with the polarization of TAMs into the M2 phenotype reveals that the polarization process is blocked indirectly. A previous investigation ${ }^{93}$ has shown that osthole not only decreases the number of M2-TAMs but also reduces the mRNA expression of TGF-b, CCL22, and MRC1 in the spleens of mice with pancreatic cancer; F4/ 80pCD206p CD11bp cells were also decreased according to flow cytometry, and these results indicate that osthole treatment could inhibit M2 polarization.

Pretreatment with pterostilbene in the NSCLC cell line THP-1-H441 co-incubation system decreased the mRNA levels of MUC-1 and NF- $\mathrm{KB}$, both of which are key molecules in promoting the polarization of M2 macrophages. ${ }^{45,109,110}$ Therefore, pterostilbene has the ability to prevent macrophages from differentiating into the M2 subtype.

CD206 is a marker of M2 macrophages, and the expression of CD206 is significantly decreased by G-RH2 compared with control conditions, which suggests that G-Rh2 could block the polarization of TAMs into the M2 phenotype. $^{94}$

Because reactive oxygen species (ROS) could influence the polarization of TAMs into the M2 phenotype, berberine treatment affected the M2 polarization process by reducing the mRNA levels of NADPH oxidase2 (NOX2) in Apc (min/+) mice. ${ }^{111,112}$

Five TCM herbs, including Gancao (Glycyrrhiza Radix et Rhizoma), Renshen (Ginseng Radix et Rhizoma), Dongchongxiacao (Cordyceps), and Ciwujia (Acanthopanacis senticosi Radix et Rhizoma Seu caulis), have the capacity to decrease the expression of arg-1 mRNA, a known M2 marker. ${ }^{48}$

This evidence shows that TCM treatment could reduce the M2-TAM phenotype by decreasing the expression levels of associated markers in tumor cells. The reduced M2-TAM phenotype may induce apoptosis or polarization to the M1-TAM phenotype, which is accompanied by increased expression of the M1-TAM phenotype marker.

\section{TCM Suppresses the Abilities and Functions of M2-TAMs}

M2-TAMs are an important TME component related to its protumoral effects, such as increased proliferation, invasion, and migration of carcinoma cells. TCM treatment inhibits the progression of tumors by suppressing the effects and functions of M2-TAMs in tumors (Figure 3).

Lin et al used flow cytometric analysis and found that the population of tumor-infiltrating macrophages was reduced in the tumors of mice treated with osthole, which indicated that osthole could block the infiltration of M2-TAMs. ${ }^{95}$

Zhao et al showed that the IC50 of baicalein for M2TAMs at $24 \mathrm{~h}, 48 \mathrm{~h}$ and $72 \mathrm{~h}$ was 191.5/107.1/41.78 $\mu \mathrm{mol} /$ $\mathrm{L}$, which indicated that baicalein could inhibit the viability of TAMs dose-dependently and time-dependently. ${ }^{49}$

As mentioned above, Huang et al used flow cytometric analysis $^{45}$ to observe that CD133-positive cell populations were increased in the NSCLC cell lines A549 and H441 after co-culture with M2-TAMs. In addition, the expression level of CD133, which is related to stemness, was increased after co-culture with M2-TAMs, which indicated that M2-TAMs could promote the generation of NSCLC stem cells. However, flow cytometric analysis also found that pterostilbene treatment inhibited the percentage of CD133-positive H441 cells in a dose-dependent manner when co-incubated with M2-polarized macrophages. Additionally, pterostilbene could decrease the selfrenewal ability of cancer cells when co-incubated with M2-TAMs.

Co-incubation with M2-TAMs significantly increased the expression level of TGF- $\beta 1$, an active EMT inducer, in 
MDA-MB-231 cells. However, pretreatment with baicalein could reverse this effect, demonstrating that baicalein suppresses increased EMT induced by co-incubation with M2-TAMs in breast cancer cells. In addition, the breast cancer cell line MDA-MB-231 can promote tumor growth and lung metastasis better after co-culture with M2-TAMs, but this effect is inhibited by baicalein, which subsequently exerts an anti-tumor effect. ${ }^{49}$

To prove the role of berberine in the invasion and migration of cancer cells, Piao et al induced M2 polarization by berberine. Finally, the migration of HT-29 cells was inhibited after co-incubation with berberine-induced M2 macrophages compared with that after co-incubation with IL-4-induced M2-TAMs. ${ }^{111}$

\section{TCM Reverses the M2 Phenotype to the MI Phenotype}

TCM could regulate the expression level of M1 phenotype- or M2 phenotype-specific markers to induce the polarization of macrophages from the M2 phenotype to the M1 phenotype (Figure 3). Baicalein treatment was demonstrated to decrease the expression level of an M2TAM phenotype-specific marker (CD206) and increase the expression level of an M1-TAM phenotype-specific marker (CD86).

Moreover, baicalein treatment decreased the mRNA expression of M2-associated cytokines (such as TGF- $\beta 1$, Arg1, and IL-10) and increased the mRNA expression of M1-associated cytokines (such as IL-12 and TNF- $\alpha$ ). In summary, baicalein not only reversed the M2 phenotype to the M1 phenotype but also led to a functional change in TAMs.

$\mathrm{Li}$ et al found that treating M2 macrophages with $\mathrm{G}-\mathrm{Rh} 2$ resulted in downregulation of the expression of the M2 marker CD206 and upregulation of the expression of the M1 marker CD16/32. Additionally, G-Rh2 treatment of human THP-1 cell-differentiated M2 macrophages resulted in the same finding. ${ }^{94}$ These results suggest that G-Rh2 has the promising ability to change the phenotype of TAMs from M2 to M1.

Piao et al used real-time PCR to observe that berberine could induce macrophage polarization from the M2 to M1 phenotype, as it was observed that the mRNA level of an M2-TAM phenotype-associated marker (IL-12) was downregulated, while IFN (a marker of the M1-TAM phenotype) was remarkably upregulated in intestinal tumors. ${ }^{111}$ In addition, berberine treatment decreased the expression of COX-2 pathway molecules to polarize the M2 phenotype to the M1 phenotype during inflammation. ${ }^{111,113,114}$ Evidence has shown that berberine can induce M2 to M1 phenotype switching and consequently affect the biological roles of TAMs. Not only extracts of TCM, but also formulae could reverse the phenotype of TAMs from M2 to M1, such as Fuzheng Jiedu Formula (FZJD), which decrease the expression of IL-10 and TGF- $\beta$ and increase the ratio of $\mathrm{M} 1 / \mathrm{M} 2 .^{87}$

Previous investigations have shown a correlation between the IL-4-STAT6 axis and M2 macrophages, while $\mathrm{C} / \mathrm{EBPb}$ promotes $\mathrm{M} 2$ polarization as an intracellular signaling molecule. Osthole has been shown to decrease $\mathrm{C} / \mathrm{EBPb}$ expression and suppress IL-4-mediated STAT6 phosphorylation to inhibit M2 macrophage activation and block M2 polarization. $^{93}$

This evidence demonstrates that TCM treatment may reverse the M2-TAM phenotype to the M1-TAM phenotype by inhibiting the expression of related signaling pathways or suppressing significant phosphorylation. TCM treatment is promising for changing the functional effects of TAMs by reversing the M2 phenotype to the M1 phenotype. The overall effects of TCM treatment on both cancer cells and TAMs are listed in Tables 1 and 2.

\section{Summary}

Previous studies have shown that traditional Chinese medicine (TCM) could help inhibit the progression of tumors, but the cells or pathways involved remain uncertain. Recent investigations suggest that tumor-associated macrophages (TAMs), particularly M2-polarized TAMs, might be potential targets for TCM treatment in cancer. TCMs have the capacity to increase the polarization of M1-TAMs, reduce the expression level of M2-TAM phenotype markers, suppress the function of the M2-TAM phenotype, block M2-TAM phenotype polarization and convert the M2 phenotype of TAMs to the M1 phenotype of TAMs in the TME, which lead to the suppression of M2-TAM function. Above all, it is worth further investigation to determine the correlation and biochemical mechanism of different TCM herb catalogues in regulating TAM polarization phenotypes and to examine the effects of multiple components in different TCM herbs that contribute to their anti-tumor effects. Although studies have shown that TCM has the potential to suppress tumor growth and reverse the polarized phenotype of TAMs, the interplay between TCM treatment and the polarization of TAMs in tumors is not fully clear. Promising and novel 
Table I Traditional Chinese Medicine (TCM) Bioactive Ingredients Have Effects on Both Cancer Cells and TumorAssociated Macrophages (TAMs)

\begin{tabular}{|c|c|c|c|}
\hline $\begin{array}{l}\text { Bioactive } \\
\text { Ingredient } \\
\text { of TCM }\end{array}$ & $\begin{array}{l}\text { Effects on } \\
\text { Cancer Cells }\end{array}$ & Effects on TAMs & Reference \\
\hline Baicalein & $\begin{array}{l}\text { Inhibits growth } \\
\text { and metastasis } \\
\text { of breast cancer } \\
\text { cells }\end{array}$ & $\begin{array}{l}\text { Induces } \\
\text { phenotype and } \\
\text { function skewing } \\
\text { of M2 } \\
\text { macrophages to } \\
\text { MI macrophages }\end{array}$ & 49 \\
\hline \multirow[t]{2}{*}{ Berberine } & $\begin{array}{l}\text { Suppresses } \\
\text { invasion and } \\
\text { migration of } \\
\text { tumor cells }\end{array}$ & $\begin{array}{l}\text { Induces } M 2 \text { to } M 1 \\
\text { phenotype } \\
\text { switching }\end{array}$ & 111 \\
\hline & $\begin{array}{l}\text { Inhibits the } \\
\text { proliferation of } \\
\text { tumor cells }\end{array}$ & & 112 \\
\hline $\begin{array}{l}\text { Ginsenoside } \\
\text { Rh2 (G-Rh2) }\end{array}$ & $\begin{array}{l}\text { Inhibits the } \\
\text { proliferation and } \\
\text { migration of } \\
\text { lung cancer cells }\end{array}$ & $\begin{array}{l}\text { Reverses the } \\
\text { phenotype of M2 } \\
\text { macrophages to } \\
\text { MI macrophages }\end{array}$ & 94 \\
\hline Osthole & $\begin{array}{l}\text { Inhibits } \\
\text { proliferation and } \\
\text { migration and } \\
\text { induces } \\
\text { apoptosis in } \\
\text { pancreatic } \\
\text { cancer cells }\end{array}$ & $\begin{array}{l}\text { Blocks the } \\
\text { infiltration of M2 } \\
\text { macrophages, } \\
\text { decreases the } \\
\text { population of M2 } \\
\text { macrophages, } \\
\text { inhibits } \\
\text { polarization into } \\
\text { M2 macrophages }\end{array}$ & 93 \\
\hline Pterostilbene & $\begin{array}{l}\text { Decreases } \\
\text { stemness and } \\
\text { self-renewal } \\
\text { ability and } \\
\text { increases the } \\
\text { apoptosis of } \\
\text { NSCLC cells }\end{array}$ & $\begin{array}{l}\text { Reduces the } \\
\text { induction of } \\
\text { cancer cell } \\
\text { stemness by M2- } \\
\text { TAMs and } \\
\text { prevents M2-TAM } \\
\text { polarization }\end{array}$ & 45 \\
\hline
\end{tabular}

cancer therapies in which TCMs target polarized TAMs could be generated after understanding the mechanisms through which TCMs affect tumors.

\section{Acknowledgments}

This work is supported in part by the National Natural Science Foundation of China (grant number 81873137 and 81603464), The Action Plan of Shanghai Municipality for
Table 2 Classical Formulae Have Effects on Both Cancer Cells and Tumor-Associated Macrophages (TAMs)

\begin{tabular}{|l|l|l|l|}
\hline $\begin{array}{l}\text { Classic } \\
\text { Formula }\end{array}$ & $\begin{array}{l}\text { Effects on } \\
\text { Cancer Cells }\end{array}$ & Effects on TAMs & Reference \\
\hline $\begin{array}{l}\text { Bu-Fei } \\
\text { (BFD) }\end{array}$ & $\begin{array}{l}\text { Suppresses the } \\
\text { proliferation, } \\
\text { migration and } \\
\text { invasion of } \\
\text { NSCLC cells }\end{array}$ & $\begin{array}{l}\text { Suppresses the } \\
\text { function of M2- } \\
\text { polarized TAMs in } \\
\text { increasing the } \\
\text { proliferation, } \\
\text { migration and } \\
\text { invasion of } \\
\text { NSCLC cells }\end{array}$ & 46 \\
\hline PHY906 & $\begin{array}{l}\text { Increases } \\
\text { apoptosis in } \\
\text { HepG2 tumors } \\
\text { treated with } \\
\text { sorafenib }\end{array}$ & $\begin{array}{l}\text { Assists sorafenib } \\
\text { in increasing both } \\
\text { macrophage } \\
\text { infiltration and the } \\
\text { MI/M2 (tumor } \\
\text { rejection) } \\
\text { signature } \\
\text { expression } \\
\text { pattern in tumor } \\
\text { cells }\end{array}$ & 92 \\
\hline
\end{tabular}

Further Accelerating the Development of Traditional Chinese Medicine (grant number ZY(2018-2020)-RCPY -2016). This study is also partially supported by Outstanding Youth Medical Talents of Shanghai "Rising Stars of Medical Talent" Youth Development Program, Academic Leader of "315" Health and Family Planning Commission System Project in Putuo District, Shanghai (grant number18Q-RC-01) and Shanghai Rising-Star Program (grant number 17QA1403400).

\section{Disclosure}

The authors report no conflicts of interest in this work.

\section{References}

1. Li J, Lin H-S. Integrative medicine: a characteristic China model for cancer treatment. Chin J Integr Med. 2011;17(4):243-245. doi:10.1007/s11655-011-0712-4

2. Li J, Li L, Liu R, Lin H-S. Establishing Chinese medicine characteristic tumour response evaluation system is the key to promote internationalization of Chinese medicine oncology. Chin J Integr Med. 2012;18(10):730-736. doi:10.1007/s11655-012-1254-0

3. Lu P, Su W, Miao Z-H, Niu H-R, Liu J, Hua Q-L. Effect and mechanism of ginsenoside Rg3 on postoperative life span of patients with non-small cell lung cancer. Chin J Integr Med. 2008;14(1):33-36. doi:10.1007/s11655-007-9002-6

4. Wang B, Cui J. Treatment of mid-late stage NSCLC using sodium cantharidinate/vitamin B6/GP regimen in clinic. $J$ Cancer Res Ther. 2014;10(5):79-81. doi:10.4103/0973-1482.139771 
5. Guo L, Bai S-P, Zhao L, Wang X-H. Astragalus polysaccharide injection integrated with vinorelbine and cisplatin for patients with advanced non-small cell lung cancer: effects on quality of life and survival. Med Oncol. 2012;29(3):1656-1662. doi:10.1007/s12032-011-0068-9

6. Ruan LW, Deng YC. [Study on effect of Xiaoaiping in enhancing efficacy of neoadjuvant chemotherapy for breast cancer and its mechanism]. Zhongguo Zhong Yao Za Zhi. 2015;40(4):749-752.

7. Liu Y, Jia ZW, Dong L, Wang R, Qiu GQ. A randomized pilot study of atractylenolide I on gastric cancer cachexia patients. Evid Based Complement Alternat Med. 2008;5(3):337-344. doi:10.1093/ecam/nem031

8. Zhong C, Li H-D, Liu D-Y, et al. Clinical study of hepatectomy combined with Jianpi Huayu Therapy for hepatocellular carcinoma. Asian Pac J Cancer Prev. 2014;15(14):5951-5957. doi:10.7314/APJCP.2014.15.14.5951

9. Qin T-J, Zhao X-H, Yun J, Zhang L-X, Ruan Z-P, Pan B-R. Efficacy and safety of gemcitabine-oxaliplatin combined with huachansu in patients with advanced gallbladder carcinoma. World J Gastroenterol. 2008;14(33):5210-5216. doi:10.3748/ wjg. 14.5210

10. James MI, Iwuji C, Irving G, et al. Curcumin inhibits cancer stem cell phenotypes in ex vivo models of colorectal liver metastases, and is clinically safe and tolerable in combination with FOLFOX chemotherapy. Cancer Lett. 2015;364(2):135-141. doi:10.1016/j. canlet.2015.05.005

11. Lu D-R, Li D-Y, Chen X-Y, Ye P-Z, Tian S-D. Clinical research of compound Zhebei granules for increasing the therapeutic effect of chemotherapy in refractory acute leukemia patients. J Tradit Chin Med. 2009;29(3):190-194. doi:10.1016/S0254-6272(09) 60063-7

12. Xu L, Feng J-M, Li J-X, et al. Tanshinone-1 induces tumour cell killing, enhanced by inhibition of secondary activation of signaling networks. Cell Death Dis. 2013;4(11):e905-e905. doi:10.1038/cddis.2013.443

13. Jia Y, Guan Q, Guo Y, Du C. Reduction of inflammatory hyperplasia in the intestine in colon cancer-prone mice by water-extract of Cistanche deserticola. Phytother Res. 2012;26(6):812-819. doi:10.1002/ptr.v26.6

14. Noy R, Pollard JW. Tumour-associated macrophages: from mechanisms to therapy. Immunity. 2014;41:49-61. doi:10.1016/j. immuni.2014.06.010

15. Steidl C, Lee T, Shah SP, et al. Tumour-associated macrophages and survival in classic Hodgkin's lymphoma. $N$ Engl $J$ Med. 2010;362:85-87. doi:10.1056/NEJMoa0905680

16. Pollard JW. Tumour-educated macrophages promote tumour progression and metastasis. Nat Rev Cancer. 2004;4(1):71-78. doi:10.1038/nrc1256

17. Sica A, Schioppa T, Mantovani A, Allavena P. Tumour-associated macrophages are a distinct M2 polarised population promoting tumour progression: potential targets of anti-cancer therapy. Eur $J$ Cancer. 2006;42(60):717-727. doi:10.1016/j.ejca.2006.01.003

18. Zheng $\mathrm{X}$, Turkowski $\mathrm{K}$, Mora J, et al. Redirecting tumour-associated macrophages to become tumouricidal effectors as a novel strategy for cancer therapy. Oncotarget. 2017;8 (29):48436-48452. doi:10.18632/oncotarget.17061

19. Hui L, Chen Y. Tumour microenvironment: sanctuary of the devil. Cancer Lett. 2015;368:7-13. doi:10.1016/j. canlet.2015.07.039

20. Chanmee T, Ontong P, Konno K, Itano N. Tumourassociated macrophages as major players in the tumour microenvironment. Cancers (Basel). 2014;6:70-90. doi:10.3390/cancers6031670

21. Ruffell B, Coussens LM. Macrophages and Therapeutic Resistance in Cancer. Cancer Cell. 2015;27:62-72. doi:10.1016/ j.ccell.2015.02.015
22. Mantovani A, Locati M. Tumour-associated macrophages as a paradigm of macrophage plasticity, diversity, and polarization. Arterioscler Thromb Vasc Biol. 2013;33:78-83. doi:10.1161/ ATVBAHA.113.300168

23. Traves PG, Luque A, Hortelano S. Macrophages, inflammation, and tumour suppressors: ARF, a new player in the game. Mediators Inflamm. 2012;10:1-11.

24. Sica A, Mantovani A. Macrophage plasticity and polarization: in vivo veritas. J Clinl Investig. 2012;122(3):787-795. doi:10.1172/JCI59643

25. Martin Brown J, Recht L, Strober S. The promise of targeting macrophages in cancer therapy. Clin Cancer Res. 2017;23 (13):3241-3250. doi:10.1158/1078-0432.CCR-16-3122

26. Zhang J, Cao J, Ma S, et al. Tumour hypoxia enhances non-small cell lung cancer metastasis by selectively promoting macrophage M2 polarization through the activation of ERK signaling. Oncotarget. 2014;5:9664-9677. doi:10.18632/oncotarget.1856

27. Pyonteck SM, Akkari L, Schuhmacher AJ, et al. CSF-1R inhibition alters macrophage polarization and blocks glioma progression. Nat Med. 2013;19:1264-1272. doi:10.1038/nm.3337

28. Mantovani A, Sica A, Sozzani S, Allavena P, Vecchi A, Locati M. The chemokine system in diverse forms of macrophage activation and polarization. Trends Immunol. 2004;25(12):677-686. doi:10.1016/j.it.2004.09.015

29. Covarrubias AJ, Aksoylar HI, Horng T. Control of macrophage metabolism and activation by mTOR and Akt signaling. Semin Immunol. 2015;27(4):286-296. doi:10.1016/j.smim.2015.08.001

30. Italiani P, Boraschi D. From monocytes to M1/M2 macrophages: phenotypical vs. functional differentiation. Front Immunol. 2014;5:514. doi:10.3389/fimmu.2014.00514

31. Hume DA. The many alternative faces of macrophage activation. Front Immunol. 2015;22(6):370-378.

32. Mills CD, Harris RA, Ley K. Macrophage polarization: decisions that affect health. J Clin Cell Immunol. 2015;6(5):364-366. doi:10.4172/2155-9899.1000364

33. Ostuni R, Kratochvill F, Murray PJ, Natoli G. Macrophages and cancer: from mechanisms to therapeutic implications. Trends Immunol. 2015;36(4):229-239. doi:10.1016/j.it.2015.02.004

34. Soave DF, Miguel MP, Tomé FD, de Menezes LB, Nagib PRA, Celes MRN. The fate of the tumour in the hands of microenvironment: role of TAMs and mTOR pathway. Mediators Inflamm. 2016;10:1-7.

35. Solinas G, Germano G, Mantovani A, Allavena P. Tumourassociated macrophages (TAM) as major players of the cancer-related inflammation. $J$ Leukoc Biol. 2009;86 (5):1065-1073. doi:10.1189/jlb.0609385

36. Condeelis J, Pollard JW. Macrophages: obligate partners for tumour cell migration, invasion, and metastasis. Cell. 2006;124 (2):263-266. doi:10.1016/j.cell.2006.01.007

37. Mantovani A, Sica A, Allavena P, Garlanda C, Locati M. Tumour-associated macrophages and the related myeloid-derived suppressor cells as a paradigm of the diversity of macrophage activation. Hum Immunol. 2009;70(5):325-330. doi:10.1016/j.humimm.2009.02.008

38. Raes G, de Baetselier P, Noel W, Beschin A, Brombacher F, Hassanzadeh GG. Differential expression of FIZZ1 and Ym1 in alternatively versus classically activated macrophages. $J$ Leukoc Biol. 2002;71(4):597-602.

39. Nair MG, Cochrane DW, Allen JE. Macrophages in chronic type 2 inflammation have a novel phenotype characterized by the abundant expression of $\mathrm{Ym} 1$ and Fizz1 that can be partly replicated in vitro. Immunol Lett. 2003;85(2):173-180. doi:10.1016/ S0165-2478(02)00225-0

40. Teng X, Li D, Champion HC, Johns RA. FIZZ1/RELM $\alpha$, a novel hypoxia-induced mitogenic factor in lung with vasoconstrictive and angiogenic properties. Circ Res. 2003;92(10):1065-1067. doi:10.1161/01.RES.0000073999.07698.33 
41. Franklin RA, Liao W, Sarkar A, et al. The cellular and molecular origin of tumour-associated macrophages. Science. 2014;344:921-925. doi: $10.1126 /$ science. 1252510

42. Horlad H, Fujiwara Y, Takemura K, et al. Corosolic acid impairs tumour development and lung metastasis by inhibiting the immunosuppressive activity of myeloid-derived suppressor cells. Mol Nutr Food Res. 2013;57:1046-1054. doi:10.1002/ mnfr.201200610

43. Luo Y, Zhou H, Krueger J, et al. Targeting tumour-associated macrophages as a novel strategy against breast cancer. J Clinl Investig. 2006;116:2132-2141. doi:10.1172/JCI27648

44. Roth F, De La Fuente AC, Vella JL, Zoso A, Inverardi L, Serafini P. Aptamer-mediated blockade of IL4Ralpha triggers apoptosis of MDSCs and limits tumour progression. Cancer Res. 2012;72:1373-1383. doi:10.1158/0008-5472.CAN-11-2772

45. Huang W-C, Chan M-L, Chen M-J, Tsai T-H, Chen Y-J. Modulation of macrophage polarization and lung cancer cell stemness by MUC1 and development of a related small-molecule inhibitor pterostilbene. Oncotarget. 2016;7 (26):39363-39375.

46. Pang L, Han S, Jiao Y, Jiang S, Xiran H, Li. P. Bu Fei Decoction attenuates the tumour associated macrophage stimulated proliferation, migration, invasion and immunosuppression of non-small cell lung cancer, partially via IL-10 and PD-L1 regulation. Int J Oncol. 2017;51:25-38. doi:10.3892/ijo.2017.4014

47. Chen XW, Yu TJ, Zhang J, et al. CYP4A in tumour-associated macrophages promotes pre-metastatic niche formation and metastasis. Oncogene. 2017;36(35):5045-5057. doi:10.1038/onc.2017.118

48. Jiang Y-X, Chen Y, Yang Y, Chen X-X, Zhang -D-D. Screening Five Qi-Tonifying herbs on M2 phenotype macrophages. Evid Based Complement Alternat Med. 2019;10:1-8.

49. Zhao X, Qu J, Liu X, et al. Baicalein suppress EMT of breas cancer by mediating tumour-associated macrophages polarization. Am J Cancer Res. 2018;8(8):1528-1540.

50. Terabe M, Matsui S, Park JM, et al. Transforming growth factor-beta production and myeloid cells are an effector mechanism through which CD1drestricted $\mathrm{T}$ cells block cytotoxic $\mathrm{T}$ lymphocyte-mediated tumour immunosurveillance: abrogation prevents tumour recurrence. J Exp Med. 2003;198:1741-1752. doi:10.1084/jem.20022227

51. Zea AH, Rodriguez PC, Atkins MB, et al. Arginaseproducing myeloid suppressor cells in renal cell carcinoma patients: a mechanism of tumour evasion. Cancer Res 2005;65:3044-3048. doi:10.1158/0008-5472.CAN-04-4505

52. Sica A, Bronte V. Altered macrophage differentiation and immune dysfunction in tumour development. J Clinl Investig. 2007;117:1155-1166. doi:10.1172/JCI31422

53. Munder M, Eichmann K, Modolell M. Alternative metabolic states in murine macrophages reflected by the nitric oxide synthase/arginase balance: competitive regulation by CD4+ $\mathrm{T}$ cells correlates with $\mathrm{Th} 1 / \mathrm{Th} 2$ phenotype. $J$ Immunol. 1998;160:5347-5354.

54. Sheng J, Chen W, Zhu HJ. The immune suppressive function of transforming growth factor-beta (TGF-beta) in human diseases. Growth Factors. 2015;33:92-101. doi:10.3109/ 08977194.2015.1010645

55. Yoshimura A, Muto G. TGF-beta function in immune suppression. Curr Top Microbiol Immunol. 2011;350:127-147.

56. Flavell RA, Sanjabi S, Wrzesinski SH, LiconaLimon P. The polarization of immune cells in the tumour ' environment by TGFß. Nat Rev Immunol. 2010;10(8):554-567. doi:10.1038/ nri2808

57. Maeda H, Shiraishi A. TGF- $\beta$ contributes to the shift toward Th2type responses through direct and IL-10- mediated pathways in tumour-bearing mice. J Immunol. 1996;156(1):73-78.
58. Liu VC, Wong LY, Jang T, et al. Tumour evasion of the immune system by converting CD4+CD25- T cells into CD4+CD25+ $\mathrm{T}$ regulatory cells: role of tumour-derived TGF-beta. J Immunol. 2007;178:2883-2892. doi:10.4049/jimmunol.178.5.2883

59. Taylor A, Verhagen J, Blaser K, Akdis M, Akdis CA. Mechanisms of immune suppression by interleukin-10 and transforming growth factor-beta: the role of $\mathrm{T}$ regulatory cells. Immunology. 2006;117:433-442. doi:10.1111/imm.2006.117. issue-4

60. $\mathrm{Xu} \mathrm{L}$, Kitani A, Strober W. Molecular mechanisms regulating TGF-beta-induced Foxp3 expression. Mucosal Immunol. 2010;3:230-238. doi:10.1038/mi.2010.7

61. Castriconi R, Cantoni C, Chiesa MD, et al. Transforming growth factor $\beta 1$ inhibits expression of NKP30 and NKG2d receptors: consequences for the NK-mediated killing of dendritic cells. Proc Natl Acad Sci U S A. 2003;100(7):4120-4125. doi:10.1073/ pnas.0730640100

62. Grutz G. New insights into the molecular mechanism of interleukin-10-mediated immunosuppression. J Leukoc Biol. 2005;77:3-15. doi:10.1189/jlb.0904484

63. Ouyang W, Rutz S, Crellin NK, Valdez PA, Hymowitz SG. Regulation and functions of the IL-10 family of cytokines in inflammation and disease. Annu Rev Immunol. 2011;29:71-109. doi:10.1146/annurev-immunol-031210-101312

64. Ruffell B, Chang-Strachan D, Chan V, et al. Macrophage IL-10 blocks CD8 + T cell-dependent responses to chemotherapy by suppressing IL-12 expression in intratumoural dendritic cells. Cancer Cell. 2014;26:623-637. doi:10.1016/j. ccell.2014.09.006

65. Beissert S, Hosoi J, Grabbe S, Asahina A, Granstein RD. IL-10 inhibits tumour antigen presentation by epidermal antigen-presenting cells. J Immunol. 1995;154(3):1280-1286.

66. Sica A, Saccani A, Bottazzi B, et al. Autocrine production of IL10 mediates defective IL-12 production and NF- $\mathrm{KB}$ activation in tumour-associated macrophages. J Immunol. 2000;164 (2):762-767. doi:10.4049/jimmunol.164.2.762

67. Fremd C, Schuetz F, Sohn C, Beckhove P, Domschke C. B cell-regulated immune responses in tumour models and cancer patients. OncoImmunology. 2013;2(7):25443. doi:10.4161/ onci. 25443

68. Lindau D, Gielen P, Kroesen M, Wesseling P, Adema GJ. The immunosuppressive tumour network: myeloid-derived suppressor cells, regulatory $\mathrm{T}$ cells and natural killer $\mathrm{T}$ cells. Immunology. 2013;138(2):105-115. doi:10.1111/imm.2013.138. issue-2

69. Ostrand-Rosenberg S, Sinha P, Beury DW, Clements VK. Crosstalk between myeloid-derived suppressor cells (MDSC), macrophages, and dendritic cells enhances tumour-induced immune suppression. Semin Cancer Biol. 2012;22(4):275-281. doi:10.1016/j.semcancer.2012.01.011

70. Landskron G, de La Fuente M, Thuwajit P, Thuwajit C, Hermoso MA. Chronic inflammation and cytokines in the tumour microenvironment. J Immunol Res. 2014;14:1-19. doi:10.1155/ 2014/149185

71. Bronte V, Zanovello P. Regulation of immune responses by L-arginine metabolism. Nat Rev Immunol. 2005;5:641-654. doi:10.1038/nri1668

72. Gallina G, Dolcetti L, Serafini P, et al. Tumours induce a subset of inflammatory monocytes with immunosuppressive activity on $\mathrm{CD} 8+\mathrm{T}$ cells. $J$ Clinl Investig. 2006;116 :2777-2790. doi:10.1172/JCI28828

73. Rodriguez PC, Ochoa AC. Arginine regulation by myeloid derived suppressor cells and tolerance in cancer: mechanisms and therapeutic perspectives. Immunol Rev. 2008;222:180-191. doi:10.1111/j.1600-065X.2008.00608.x 
74. Rodr'1guez PC, Ochoa AC. Arginine regulation by myeloid derived suppressor cells and tolerance in cancer: mechanisms and therapeutic perspectives. Immunol Rev. 2008;222 (1):180-191. doi:10.1111/j.1600-065X.2008.00608.x

75. Bak SP, Alonso A, Turk MJ, Berwin B. Murine ovarian cancer vascular leukocytes require arginase-1 activity for $\mathrm{T}$ cell suppression. Mol Immunol. 2008;46(2):258-268. doi:10.1016/j. molimm.2008.08.266

76. Chang CI, Liao JC, Kuo L. Macrophage arginase promotes tumour cell growth and suppresses nitric oxidemediated tumour cytotoxicity. Cancer Res. 2001;61(3):1100-1106.

77. Wyckoff JB, Wang Y, Lin EY, et al. Direct visualization of macrophage-assisted tumour cell intravasation in mammary tumours. Cancer Res. 2007;67:2649-2656. doi:10.1158/00085472.CAN-06-1823

78. Hagemann T, Wilson J, Burke F, et al. Ovarian cancer cells polarize macrophages toward a tumour-associated phenotype. $J$ Immunol. 2006;176(8):5023-5032. doi:10.4049/jimmunol. 176.8 .5023

79. Giraudo E, Inoue M, Hanahan D. An amino-bisphosphonate targets MMP-9-expressing macrophages and angiogenesis to impair cervical carcinogenesis. J Clinl Investig. 2004;114 (5):623-633. doi:10.1172/JCI200422087

80. Krecicki T, Zalesska-Krecicka M, Jelen M, Szkudlarek T, Horobiowska M. Expression of type IV collagen and matrix metalloproteinase-2 (type IV collagenase) in relation to nodal status in laryngeal cancer. Clin Otolaryngol Allied Sci. 2001;26 (6):469-472. doi:10.1046/j.0307-7772.2001.00503.x

81. Makitie T, Summanen P, Tarkkanen A, Kivel T. Tumourinfiltrating macrophages (CD68+ cells) and prognosis in malignant uveal melanoma. Invest Ophthalmol Vis Sci. 2001;42 (7):1414-1421.

82. Nishie A, Ono M, Shono T, et al. Macrophage infiltration and heme oxygenase-1 expression correlate with angiogenesis in human gliomas. Clin Cancer Res. 1999;5(5):1107-1113.

83. Koide N, Nishio A, Sato T, Sugiyama A, Miyagawa SI. Significance of macrophage chemoattractant protein1 expression and macrophage infiltration in squamous cell carcinoma of the esophagus. Am J Gastroenterol. 2004;99(9):1667-1674. doi:10.1111/j.1572-0241.2004.30733.x

84. Hanada T, Nakagawa M, Emoto A, Nomura T, Nasu N, Nomura Y. Prognostic value of tumour-associated macrophage count in human bladder cancer. Int J Urol. 2000;7(7):263-269. doi:10.1046/j.1442-2042.2000.00190.x

85. Lissbrant IF, Stattin P, Wikstrom P, Damber JE, Egevad L, Bergh A. Tumour associated macrophages in human prostate cancer: relation to clinicopathological variables and survival. Int J Oncol. 2000;17(3):445-451. doi:10.3892/ijo.17.3.445

86. Leek RD, Lewis CE, Whitehouse R, Greenall M, Clarke J, Harris AL. Association of macrophage infiltration with angiogenesis and prognosis in invasive breast carcinoma. Cancer Res. 1996;56(20):4625-4629.

87. Guo Q, Li J, Lin H. Effect and molecular mechanisms of traditional Chinese medicine on regulating tumour immunosuppressive microenvironment. Biomed Res Int. 2015;10:1-12.

88. Liu CY, Xu JY, Shi XY, et al. M2-polarized tumour-associated macrophages promoted epithelial-mesenchymal transition in pancreatic cancer cells, partially through TLR4/IL-10 signaling pathway. Lab Investig. 2013;93:844-854. doi:10.1038/ labinvest.2013.69

89. Ravi J, Elbaz M, Wani NA, Nasser MW, Ganju RK. Cannabinoid receptor-2 agonist inhibits macrophage induced EMT in non-small cell lung cancer by downregulation of EGFR pathway. Mol Carcinog. 2016;55:2063-2076. doi:10.1002/mc. v55.12
90. Schoppmann SF. Lymphangiogenesis, inflammation and metastasis. Anticancer Res. 2005;25(6):4503-4511.

91. Lin X, Yi Z, Diao J, et al. ShaoYao decoction ameliorates colitis-associated colorectal cancer by downregulating proinflammatory cytokines and promoting epithelial-mesenchymal transition. J Transl Med. 2014;12(1):105. doi:10.1186/1479-5876-12-105

92. Lam W, Jiang Z, Guan F, et al. PHY906 (KD018), an adjuvant based on a 1800-year-old Chinese medicine, enhanced the anti-tumour activity of Sorafenib by changing the tumour microenvironment. Sci Rep. 2015;5:9384. doi:10.1038/srep09384

93. Wang B, Zheng X, Liu J, et al. Osthole inhibits pancreatic cancer progression by directly exerting negative effects on cancer cells and attenuating tumour-infiltrating M2 macrophages. J Pharmacol Sci. 2018;137:290-298. doi:10.1016/j.jphs.2018.07.007

94. Li H, Huang N, Zhu W, et al. Modulation the crosstalk between tumourassociated macrophages and non-small cell lung cancer to inhibit tumour migration and invasion by ginsenoside $\mathrm{Rh} 2 . B M C$ Cancer. 2018;18(579). doi:10.1186/s12885-018-4242-8

95. Lin J, Li Q, Chen H, Lin H, Lai Z, Peng J. Hedyotis diffusa Willd. extract suppresses proliferation and induces apoptosis via IL-6-inducible STAT3 pathway inactivation in human colorectal cancer cells. Oncol Lett. 2015;9:1962-1970. doi:10.3892/ ol.2015.2956

96. Liu J, Zhang J, Huang L, Zhu X, Chen W, Hu. P. XuefuZhuyu Tang exerts antitumour effects by inhibiting glioma cell metastasis and invasion via regulating tumour microenvironment. Onco Targets Ther. 2016;9:3603-3612. doi:10.2147/OTT.S104108

97. Ostuni R, Kratochvill F, Murray PJ, Natoli G. Macrophages and cancer: from mechanisms to therapeutic implications. Trends Immunol. 2015;36:229-239. doi:10.1016/j.it.2015.02.004

98. Kessenbrock K, Wang CY, Werb Z. Matrix metalloproteinases in stem cell regulation and cancer. Matrix Biol. 2015;44-46:184-190. doi:10.1016/j.matbio.2015.01.022

99. Brown GT, Murray GI. Current mechanistic insights into the roles of matrix metalloproteinases in tumour invasion and metastasis. J Pathol. 2015;237(3):273-281. doi:10.1002/path.4586

100. Comunanza V, Corà D, Orso F, et al. VEGF blockade enhances the antitumour effect of BRAFV600E inhibition. EMBO Mol Med. 2017;9(2):219-237. doi:10.15252/emmm.201505774

101. Guo Q, Jie L, Lin H. Effect and molecular mechanisms of traditional Chinese medicine on regulating tumour immunosuppressive microenvironment. Biomed Res Int. 2015;261:1-12.

102. Li Y-L, Sun B-G, Xiang T, Chen Z-X, Zhang S-J. Effect of invigorating spleen and detoxification decoction on $\mathrm{MHCI} /$ MHCII in spleen-deficiency liver cancer rats survival. J Chin Med Mater. 2014;37(3):456-462.

103. Villa-Morales M, Fernandez-Piqueras J. Targeting the Fas/FasL signaling pathway in cancer therapy. Expert Opin Ther Targets. 2012;16(1):85-101. doi:10.1517/14728222.2011.628937

104. Chang Y, Zhao Y, Zhan H, Wei X, Liu T, Zheng B. Bufalin inhibits the differentiation and proliferation of human osteosarcoma cell line hMG63-derived cancer stem cells. Tumour Biol. 2014;35(2):1075-1082. doi:10.1007/s13277-013-1143-y

105. Pan HC, Lai DW, Lan KH, et al. Honokiol thwarts gastric tumour growth and peritoneal dissemination by inhibiting Tpl2 in an orthotopic model. Carcinogenesis. 2013;34(11):2568-2579. doi:10.1093/carcin/bgt243

106. Liu C-P, Zhang X, Tan Q-L, et al. NF- $\kappa B$ pathways are involved in M1 polarization of RAW 264.7 macrophage by polyporus polysaccharide in the tumour microenvironment. PLoS One. 2017;12(11):317.

107. Xu F, Cui W, Zhao Z, et al. Targeting tumour microenvironment: effects of Chinese herbal formulae on macrophage-mediated lung cancer in mice. Evid Based Complement Alternat Med. 2017;2017:716-718. doi:10.1155/2017/7187168 
108. Elton TS, Selemon H, Elton SM, Parinandi NL. Regulation of the MIR155 host gene in physiological and pathological processes. Gene. 2013;532(1):1-12. doi:10.1016/j.gene.2012.12.009

109. Chang CP, Su YC, Lee PH, Lei HY. Targeting NF-kB by autophagy to polarize hepatoma-associated macrophage differentiation. Autophagy. 2013;9:619-621. doi:10.4161/auto.23546

110. Saccani A, Schioppa T, Porta C, et al. p50 nuclear factor-kappaB overexpression in tumour-associated macrophages inhibits M1 inflammatory responses and antitumour resistance. Cancer Res. 2006;66:11432-11440. doi:10.1158/0008-5472.CAN-06-1867

111. Piao M, Cao H, He N, et al. Berberine inhibits intestinal polyps growth in Apc $(\mathrm{min} /+)$ mice via regulation of macrophage polarization. Evid Based Complement Alternat Med. 2016;10:1-8. doi:10.1155/2016/5137505
112. Dandan L, Zhang Y, Liu K, et al. Berberine inhibits colitis-associated tumourigenesis via suppressing inflammatory responses and the consequent EGFR signaling-involved tumour cell growth. Lab Investig. 2017;97:1343-1353. doi:10.1038/ labinvest.2017.71

113. Cheng S-E, Lee I-T, Lin -C-C, Wu W-L, Hsiao L-D, Yang C-M. ATP mediates NADPH oxidase/ROS generation and COX-2/ PGE2 expression in A549 cells: role of P2 receptordependent STAT3 activation. PLoS One. 2013;8(1):542-547.

114. Kim HG, Kim YR, Park JH, et al. Endosulfan induces COX-2 expression via NADPH oxidase and the ROS, MAPK, and Akt pathways. Arch Toxicol. 2015;89(11):2039-2050. doi:10.1007/ s00204-014-1359-7

\section{Publish your work in this journal}

Drug Design, Development and Therapy is an international, peerreviewed open-access journal that spans the spectrum of drug design and development through to clinical applications. Clinical outcomes, patient safety, and programs for the development and effective, safe, and sustained use of medicines are a feature of the journal, which has also been accepted for indexing on PubMed Central. The manuscript management system is completely online and includes a very quick and fair peer-review system, which is all easy to use. Visit http://www. dovepress.com/testimonials.php to read real quotes from published authors. 